\title{
History of myxozoan character evolution on the basis of rDNA and EF-2 data
}

Ivan Fiala ${ }^{1,2^{*}}$, Pavla Bartošová ${ }^{1,2}$

\begin{abstract}
Background: Phylogenetic relationships among myxosporeans based on ribosomal DNA data disagree with traditional taxonomic classification: a number of myxosporeans with very similar spore morphology are assigned to the same genera even though they are phylogenetically distantly related. The credibility of rDNA as a suitable marker for Myxozoa is uncertain and needs to be proved. Furthermore, we need to know the history of myxospore evolution to understand the great diversity of modern species.

Results: Phylogenetic analysis of elongation factor 2 supports the ribosomal DNA-based reconstruction of myxozoan evolution. We propose that SSU rDNA is a reliable marker for inferring myxozoan relationships, even though SSU rDNA analysis markedly disagrees with the current taxonomy. The analyses of character evolution of 15 morphological and 5 bionomical characters show the evolution of individual characters and uncover the main evolutionary changes in the myxosporean spore morphology and bionomy. Most bionomical and several morphological characters were found to be congruent with the phylogeny. The summary of character analyses leads to the simulation of myxozoan ancestral morphotypes and their evolution to the current species. As such, the ancestor of all myxozoans appears to have infected the renal tubules of freshwater fish, was sphaerosporid in shape, and had a spore with polar capsules that discharged slightly sideways. After the separation of Malacosporea, the spore of the common myxosporean ancestor then changed to the typical sphaerosporid morphotype. This species inhabited the marine environment as a parasite of the gall bladder of marine fish and ultimately separated into the three main myxosporean lineages evident today. Two of these lineages re-entered the freshwater environment, one as a myxosporean with Chloromyxum and another with a primitive sphaerosporid morphotype. The common ancestor of all marine myxosporeans had a ceratomyxid shape of spore.

Conclusions: We support rDNA based myxozoan phylogeny by the analysis of a protein coding gene and demonstrate the reliability of rDNA as a marker explaining myxozoan relationships. Our tracing the history of myxozoan character evolution discloses ancestral morphotypes and shows their development over the course of evolution. We point out several myxozoan characters that are to a certain extent congruent with the phylogeny and determined that the discrepancy between phylogeny and current taxonomy based on spore morphology is due to an extreme myxospore plasticity occurring during myxozoan evolution.
\end{abstract}

\section{Background}

Myxozoans are microscopic metazoan parasites with extremely reduced body size and structure. Vegetative stages form spores in both hosts (invertebrates and vertebrates) in their life cycle. The only exceptions are malacosporeans in the genus Buddenbrockia, which live in bryozoans and have macroscopic worm-like stages in their life cycles [1]. Myxozoans were thought to be

\footnotetext{
* Correspondence: fiala@paru.cas.cz

${ }^{1}$ Institute of Parasitology, Biology Centre, Academy of Sciences of the Czech Republic, Branišovská 31, 37005 České Budějovice, Czech Republic
}

protists for more than one hundred years until the 1990 s due to the simplicity of their microscopic spores. Phylogenetic analysis of the first myxozoan SSU rDNA [2] then confirmed the marginalized suppositions that Myxozoa are multicellular organisms $[3,4]$ and placed Myxozoans within the Metazoa. However, the phylogenetic position of the Myxozoa within the Metazoa was uncertain due to the weakness of SSU rDNA data [2,5-7]. More recent phylogenetic analysis based on the sequences of numerous protein-coding genes of the
C Biomed Central

๑ 2010 Fiala and Bartošová; licensee BioMed Central Ltd. This is an Open Access article distributed under the terms of the Creative Commons Attribution License (http://creativecommons.org/licenses/by/2.0), which permits unrestricted use, distribution, and reproduction in any medium, provided the original work is properly cited. 
malacosporean, B. plumatellae [8], has suggested Cnidaria as the most closely related taxon to Myxozoa.

The phylogenetic analyses of an increasing number of myxosporean SSU rDNA sequences raised doubt about the taxonomic scheme of Myxosporea [9-11]. Great discrepancies were found between the phylogenetic relationships of myxosporeans inferred from the SSU rDNA and spore-based myxosporean taxonomy. A number of myxosporean species with very different types of myxospores (hence belonging to different genera) are grouped together by the phylogenetic analysis. For example, many traditional genera, such as Henneguya, Sphaerospora, Myxidium, Zschokkella or Chloromyxum, are polyphyletic. Such incongruence between taxonomy and SSU rDNA phylogeny raises questions about the reliability of the SSU rDNA as an appropriate phylogenetic marker for Myxosporea.

Generally, phylogenetic relationships based solely on a single gene reflect the phylogeny of that particular gene and may not correspond to the true species phylogeny. Moreover, single-gene analyses do not provide sufficient resolution for some nodes or sometimes give conflicting results. This is often ascribed to the limited number of nucleotides that can be aligned or to differing rates of sequence evolution leading to long-branch attraction $[12,13]$. Therefore, the congruent results of phylogenetic analyses based on two or more molecular markers can provide a more solid ground for better taxonomic classification. Partial sequences of the large subunit ribosomal RNA (LSU rDNA) gene have served as a second molecular marker and confirmed the SSU rDNA-based relationships of the Multivalvulida [14]. Both of these genes provided similar tree topology and were also congruent with another molecular marker (HSP-70) used at the intraspecific level in the phylogeographical study of Kudoa thyrsites [15]. The recent work of Bartošová et al. [16] confirmed the similar evolution of SSU and LSU
rDNA genes for myxosporeans in general. The rDNA phylogenetic analyses of primary sequences were also confirmed by the analysis of variable parts of the secondary structure of SSU rDNA [17]. In the present study, we analyze elongation factor 2 (EF2) as the first protein-coding marker and we compare the obtained phylogeny to that of rDNA.

Evolution of myxozoan morphological characters became a big puzzle after the analyses of molecular data revealed the discrepancy between morphology and phylogeny. The myxospore is practically the only life cycle stage that provides a sufficient number of characters suitable for taxonomy and cladistics. Knowledge of the evolutionary history of myxospore characters is therefore important for taxonomic revisions necessary to correct myxozoan taxonomy. We constructed a matrix of morphological and bionomical characters of selected myxozoans whose rDNA sequences were available for phylogenetic analyses. Then, we mapped these characters on the SSU rDNA-based tree to trace the ancestral character features to study the evolution of myxozoan morphotypes as sets of particular character features.

\section{Results and Discussion}

\section{Multigene phylogenetic analyses}

Phylogenetic analysis of the EF2 amino acid sequence data of twelve myxosporeans (GenBank Acc. Nos. HM037908 - HM037919) was congruent with the rDNA-based phylogeny (Figure 1). Four of five nodes (ML, MP) with high bootstrap support in a concatenated SSU+LSU analysis were present in the EF2 tree. Surprisingly, the only exception was the non-sister relationship of two Kudoa spp. These species were also paraphyletic in additional phylogenetic analyses of nucleotide EF2 data (both with all nucleotides and excluding the third position) as well as in the analysis based on EF2 codons (additional file 1). The twelve

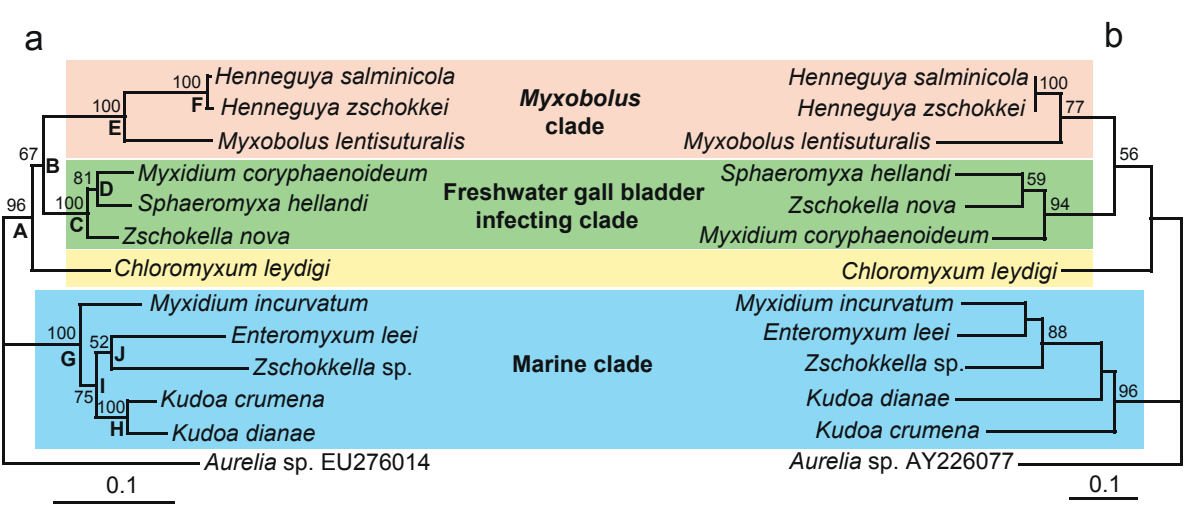

Figure 1 Comparison of rDNA and EF2 based phylogeny of myxosporeans. Maximum likelihood tree of (a) concatenated SSU + LSU rDNA data $(-\ln =14993.1047, \mathrm{GTR}+\Gamma$ model, $\alpha=0.3415)$ and (b) EF2 data $(-\ln =2973.9753$, aa rate matrix $=$ wag, $\alpha=0.4692)$. The numbers at the nodes represent bootstrap values (> 50\%). Cnidarian Aurelia sp. was set as outgroup sequence. Scale bar is given under the tree. 


\begin{tabular}{|c|c|c|c|c|c|c|c|c|c|c|c|}
\hline \multirow[t]{2}{*}{ Gene } & \multirow[t]{2}{*}{ Method } & \multicolumn{10}{|c|}{ Nodal support } \\
\hline & & $\mathrm{A}$ & B & $\mathrm{C}$ & $\mathrm{D}$ & $\mathrm{E}$ & $\mathrm{F}$ & $\mathrm{G}$ & $\mathrm{H}$ & I & $\mathrm{J}$ \\
\hline \multirow[t]{3}{*}{$\mathrm{SSU}+\mathrm{LSU}$} & BI & 1.00 & 0.99 & 1.00 & 1.00 & 1.00 & 1.00 & 1.00 & 1.00 & 0.96 & 0.73 \\
\hline & ML & 96 & 67 & 100 & 81 & 100 & 100 & 100 & 100 & 75 & 52 \\
\hline & MP & 98 & - & 100 & 83 & 100 & 100 & 100 & 100 & 87 & 88 \\
\hline \multirow[t]{3}{*}{ EF2aa } & BI & 1.00 & 1.00 & 1.00 & - & 1.00 & 1.00 & 1.00 & - & - & 0.96 \\
\hline & ML & 44 & 56 & 94 & - & 77 & 100 & 96 & - & - & - \\
\hline & MP & 55 & 44 & 100 & - & 67 & 100 & 76 & - & - & 53 \\
\hline EF2codon & ML & - & 45 & 98 & - & 72 & 100 & 98 & 32 & - & - \\
\hline \multirow[t]{3}{*}{ EF2nt-3 } & BI & - & - & 0.90 & - & - & 1.00 & 1.00 & & - & - \\
\hline & ML & - & - & 97 & - & 70 & 100 & 95 & 30 & - & - \\
\hline & MP & - & - & 100 & - & 90.00 & 100 & 94 & 48 & - & - \\
\hline \multirow[t]{3}{*}{ EF2nt } & BI & - & - & 1.00 & - & 0.95 & 1.00 & 1.00 & - & - & - \\
\hline & ML & - & - & 98 & - & 63 & 100 & 93 & - & - & - \\
\hline & MP & - & - & 97 & - & 84 & 100 & 50 & - & - & - \\
\hline $\mathrm{SSU}+\mathrm{LSU}+\mathrm{EF} 2 \mathrm{aa}$ & BI & 1.00 & 1.00 & 1.00 & - & 1.00 & 1.00 & 1.00 & 1.00 & - & 0.83 \\
\hline $\mathrm{SSU}+\mathrm{LSU}+\mathrm{EF} 2 \mathrm{nt}-3$ & BI & 1.00 & 1.00 & 1.00 & - & 1.00 & 1.00 & 1.00 & 1.00 & 0.53 & 0.80 \\
\hline $\mathrm{SSU}+\mathrm{LSU}+\mathrm{EF} 2 \mathrm{nt}$ & BI & 1.00 & 1.00 & 1.00 & - & 1.00 & 1.00 & 1.00 & 1.00 & - & - \\
\hline \multirow[t]{3}{*}{ SSU } & BI & 0.98 & 1.00 & 1.00 & 0.98 & 1.00 & 1.00 & 1.00 & 1.00 & - & 0.72 \\
\hline & ML & 66.00 & 88.00 & 90.00 & 81 & 100 & 100 & 100 & 100 & - & - \\
\hline & MP & 91 & 93 & 100 & 88 & 100 & 100 & 100 & 100 & - & 72 \\
\hline \multirow[t]{3}{*}{ LSU } & BI & 0.99 & - & 1.00 & - & 1.00 & 1.00 & 1.00 & 1.00 & 0.98 & 0.56 \\
\hline & ML & 94 & - & 100 & - & 100 & 100 & 100 & 86 & 84 & 41 \\
\hline & MP & 92 & - & 100 & - & 100 & 100 & 100 & 80 & 82 & 72 \\
\hline
\end{tabular}

Figure 2 Summary of tree nodal supports resulted from different analyses of various datasets. Nodes A - J are specified in the Figure 1. $\mathrm{ML}=$ maximum likelihood; $\mathrm{MP}=$ maximum parsimony; $\mathrm{BI}=$ Bayesian inference. $\mathrm{EF} 2 \mathrm{aa}=\mathrm{EF} 2$ amino acid sequence data; $\mathrm{EF} 2 \mathrm{codon}=\mathrm{EF} 2$ sequence data analysed as codons; EF2nt-3 = EF2 nucleotide data with exclusion of the third site; EF2nt = EF2 nucleotide data. Dashes indicate missing nodes in the resulted tree.

myxosporeans studied represent three significant phylogenetic groups as defined by Fiala [11] in the SSU rDNA-based study. All of these groups were clear in our EF2 analysis. Moreover, the position of Chloromyxum leydigi as an independent lineage was the same. Nodes with moderate or insignificant support resulting from concatenated rDNAs analysis were frequently missing in the EF2 analyses and the sub-tree topologies were different (Figure 2). These sub-tree topologies were different when either single SSU or LSU rDNA analyses were compared to their concatenated analysis (additional file 2 ). These differences apply mainly for nodes in the marine clade, which includes five species representing four subgroups with unresolved relationships in broad rDNA based analysis [16]. Reasons for this incongruence may be related to the lack of informative characters that would allow resolution of particular species relationships. Concatenated analysis of all three genes (additional file 3) did not improve the nodal supports and tree resolution (Figure 2).

Congruence of rDNA and protein-coding gene analysis support the relevance of SSU rDNA as a marker for inferring myxosporean phylogeny. We can be confident that the polyphyletic and paraphyletic relationships revealed by SSU rDNA correspond to the correct species evolution. This supports the presumption that an evolutionary origin of many morphologically similar myxospore types is the result of convergent appearance of successful spore design in myxosporean evolution as pointed out for sphaerosporids by Holzer et al. [10].

\section{Tracing of history of myxozoan character evolution}

Tracing the history of character evolution can help uncover the morphology of the ancestral myxozoan species. There are no data (and very likely there will never be) about myxozoans from the fossil record. The only possible way we can identify ancestral species is by simulating their evolution via sophisticated computer programs. We chose the Mesquite program, which is able to calculate the ancestral state of a given character by the chosen method similarly as e.g. for annelid character evolution [18]. This simulation allowed us to conceive what the ancestor of all myxozoans probably was and to find out the morphological characters of ancestors of the main clades. While the weakness of the analysis may be in the selection of available species, the general scheme of deep ancestral evolution would nevertheless not be affected.

We mapped 15 morphological and 5 bionomical characters on the SSU rDNA tree, which was constructed to cover all known phylogenetic groups of myxosporeans. The matrix includes also five unnamed, formally not characterized species, since they represent significant morphotypes and phylogenetically important taxa for the clades in which these species cluster. The summary of all characters, features, and taxa used in this analysis is shown in Figure 3. We inferred a character history for each character using likelihood ancestral state reconstruction methods to understand the process of individual character change as well as the change of the spore as a complex of characters. Figure 4 summarizes the 


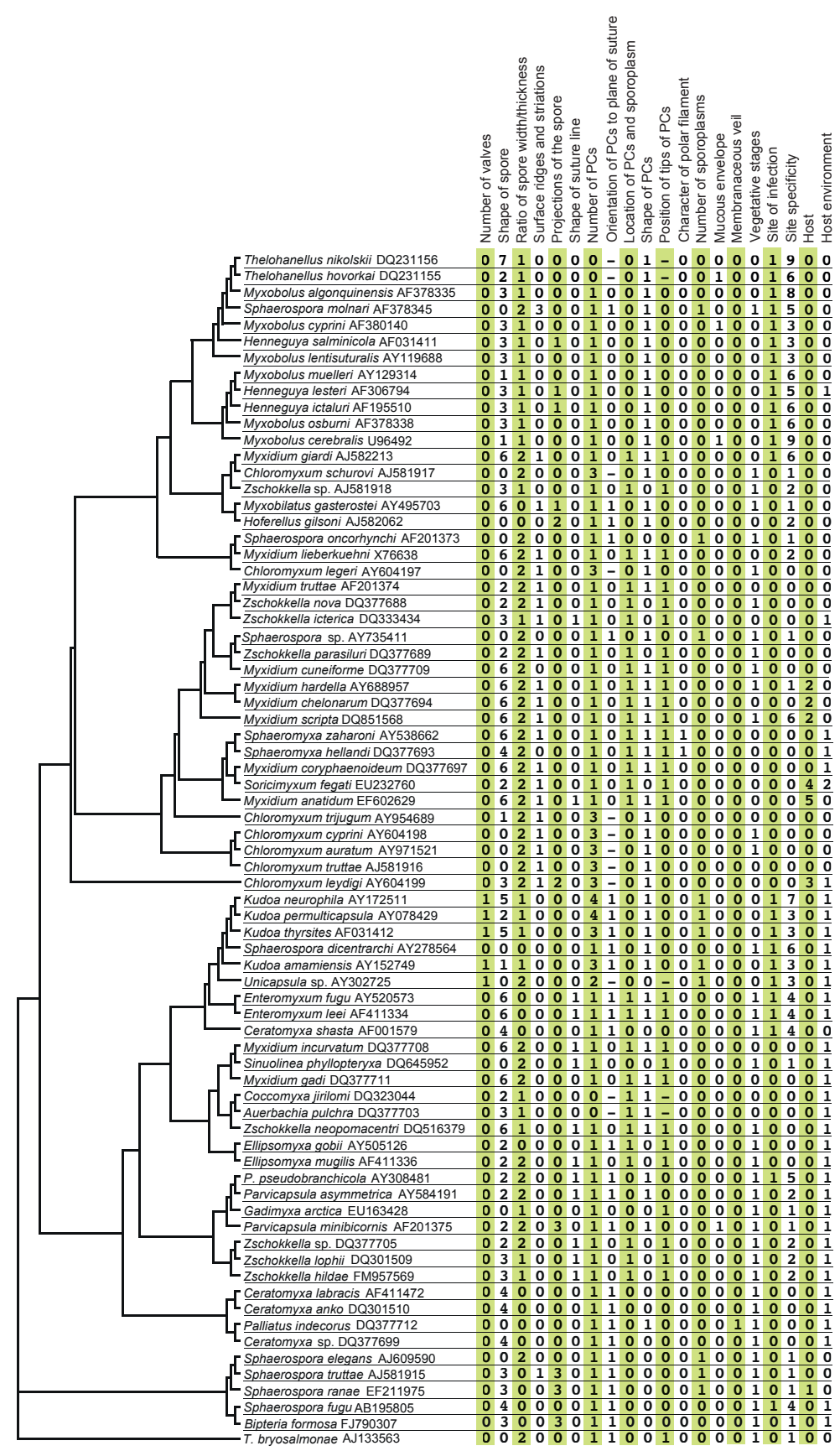

Figure 3 Matrix of myxozoan characters evaluated in the analysis of character evolution. Myxozoan ML tree based on SSU rDNA (GenBank AccNos. are behind the species names) is completed with character matrix, which is coded as follows: Number of valves: 0 - two, 1 - more than two. Shape of spore: 0 - spherical or subspherical, 1 - flattened sphere, 2 - ellipsoidal, 3 - flattened ellipsoid, 4 - banana or crescent, 4 - stellate, 5 - spindle, 6 - droplike. Ratio of dimensions of spore width to thickness: 0 - thickness larger than width, 1 - width larger than thickness, 2 - width equal to thickness. Surface ridges and striations: 0 - absent, 1 - present. Projections of the spore: 0 - no projections, 1 - caudal appendages, 2 - filamentous, 3 - bulges. Shape of suture line: 0 - straight, 1 - curved. Number of polar capsules (PCs): 0 - one, 1 - two, 2 - three, 3 - four, 4 - five and more. Orientation of PCs to a plane of the suture: 0 -PCs in the apex of the spore are set in the sutural plane, 1 - PCs are set in a plane essentially perpendicular to the suture plane. Location of PCs and sporoplasm: 0 -PCs at anterior part and sporoplasm posteriorly, 1 - PCs at opposite ends and sporoplasm in the middle. Shape of PCs: 0 - spherical or subspherical, 1 - pyriform. Position of anterior ends of PCs: 0 - convergent, 1 - divergent. Character of polar filament: 0 - tubular, 1 -flattened. Number of sporoplasms: 0 - one, 1 - two. Mucous envelope: 0 - absent, 1 - present. Membranaceous veil: 0 - absent, 1 - present. Vegetative stages: 0 -polysporic, 1 - small mono or disporic. Site of infection: 0 - coelozoic, 1 - histozoic. Site specificity: 0 - gall bladder and biliary ducts, 1 - renal tubules, 2 - urinary bladder, 3 - muscle, 4 - intestine, 5 - gills or pseudobranchs, 6 - without site specificity, 7 - nervous system, 8 - ovary, 9 - cartilage. Host: 0 - fish, 1 - amphibian, 2 - reptile, 3 - elasmobranchs, 4 - mammal, 5 - bird. Host environment: 0 - freshwater, 1 - marine, 2 - terrestrial. 


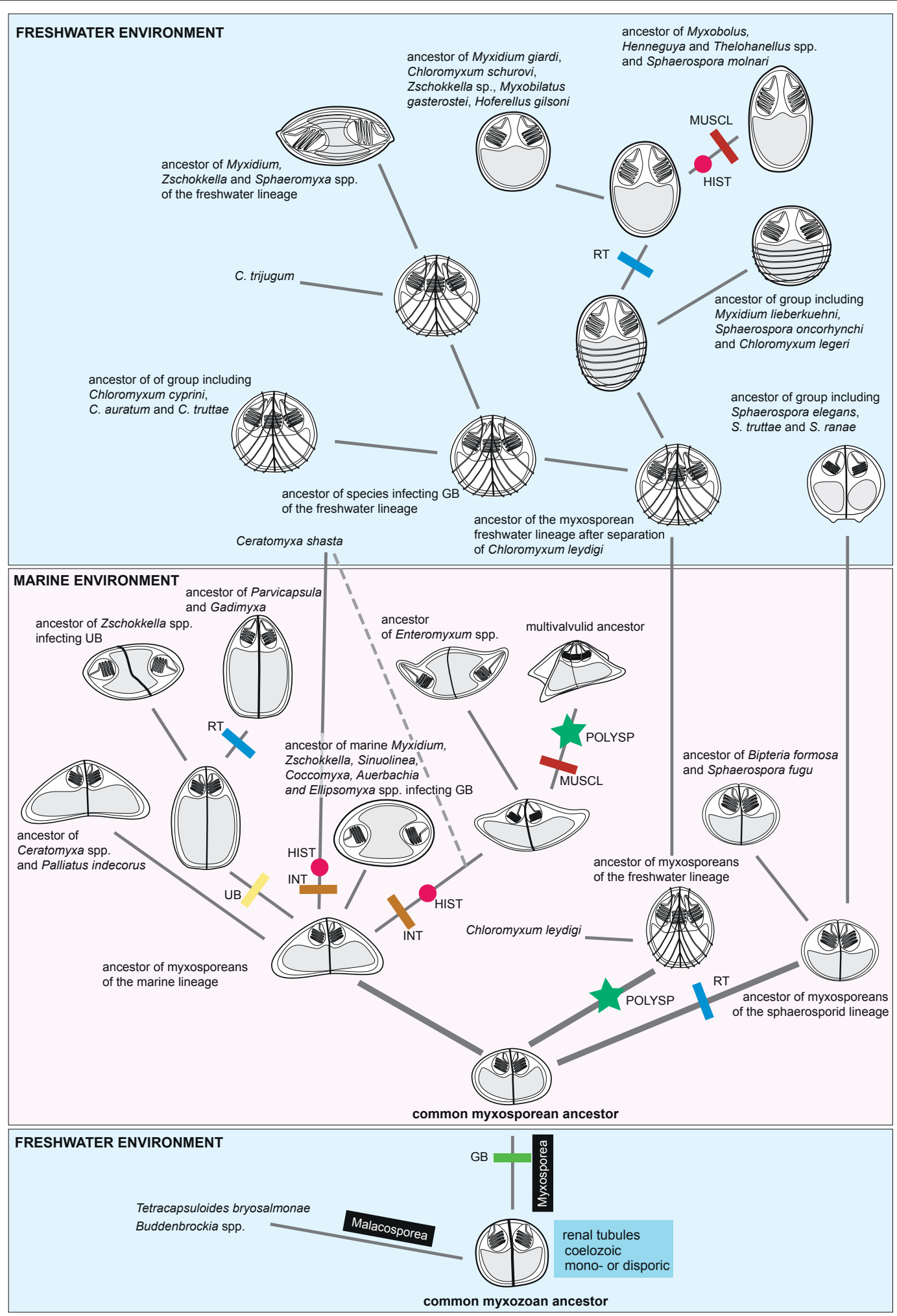

Figure 4 Hypothetical evolution of ancestral morphotypes and their bionomical characters. Schematic drawing summarises evolution of myxospore morphology based on the SSU rDNA phylogeny and character evolution analysis. Rectangles, circles and asterisks show the switches of bionomical characters. GB - gall bladder, UB - urinary bladder, RT - renal tubules, MUSCL - muscles, INT - intestine, HIST - histozoic, POLYSP polysporic. Dashed lines indicate two alternative branches leading to Ceratomyxa shasta. 
evolutionary history of myxospore morphology and the presumed switches among bionomical characters based upon this analysis. Cladograms in additional file 4 illustrate the particular character histories for all determined characters.

\section{Number of valves}

The ancestor of all myxozoans had two spore valves. The analysis showed that the change to the two-valve character state evolved only once in an ancestor in one of the marine lineages. The number of valves is the main taxonomic characteristic on which the separation of the class Myxosporea into the orders Bivalvulida Shulman, 1959 and Multivalvulida Shulman, 1959 is based. Evolution of this character makes Bivalvulida a paraphyletic group and renders the basic taxonomic division of Myxosporea erroneous.

\section{Shape of spore}

Myxosporeans have a variable spore shape that is difficult to delimitate into a few defined categories. We divided myxospores into eight basic three-dimensional categories for the purpose of our analysis (See legend in additional file $4 \mathrm{~b}$ ). The common myxozoan ancestor had a spherical spore. The myxospore changed its shape by the enlargement of its width to a crescent or bananalike shape after the switch to parasitizing marine fish. Descendants of this marine ancestor then diversified into several different spore shapes, which is in contrast to the rather uniform spore shape in ancestors of the main groups of the freshwater lineage.

\section{Ratio of dimensions of spore width to thickness}

This character is derived from the shape of the spore and uses only three features to characterize spore dimensions. Such a simple definition of spore shape facilitated the tracing of the evolution of spore shape and showed that the ratio of spore dimensions is a useful character shared in many phylogenetic groups. The analysis suggested that the ancient ancestors had spores thicker or of the same thickness as the width. Spores wider than thick appeared more recently in the myxosporean evolution.

\section{Surface ridges and striations}

The analysis showed that the primitive feature of this character is a smooth shell valve. Surface ridges then evolved in an ancestor of the freshwater lineage after the separation of myxosporeans into marine and freshwater lineages. Surface ridges are absent in all marine species except for several marine species clustering out of the marine clade, e.g. Myxidium coryphaenoideum, Sphaeromyxa zaharoni and Chloromyxum leydigi. This supports the position of Sphaeromyxa, a typical marine genus with a history of parasitism in the freshwater fishes, inside the freshwater lineage.

\section{Projections of the spore}

A typical example of spore projections are the caudal appendages on Henneguya spores. Species with this feature, distinguishing Henneguya from Myxobolus spp., do not share a common ancestor. Our analysis includes only several Myxobolus and Henneguya sequences, which does not reflect the true multiple origin of caudal appendages and the polyphyletic pattern of Henneguya spp. as described elsewhere [9,11]. Spore filaments are less frequent features that independently appeared only in the two terminal taxa under study. Spore bulges evolved in the ancestor of basal freshwater sphaerosporids and independently in Bipteria formosa, Parvicapsula minibicornis and Sphaerospora molnari.

\section{Shape of suture line}

A curved sutural line is a typical homoplastic character found several times on terminal nodes of certain Myxidium, Zschokkella, Parvicapsula, Enteromyxum and Sinuolinea spp.

\section{Number of polar capsules}

The ancestral state for the number of polar capsules (PCs) is two. After the split of Myxozoa into the two major freshwater and marine clades, the ancestor of the lineage with mostly freshwater species probably multiplied its PCs to four. A four-PC spore was then reduced twice to the two-PC spore within the evolution of this lineage. The four-PC spore then appeared independently again in the terminal nodes represented by Chloromyxum legeri and C. schurovi. Multiplication of PCs in the multivalvulids (Kudoa spp. and Unicapsula sp.) evolved along with an increase in number of spore valves. The two-PC character of spore changed twice to a spore with only one PC in myxozoan evolution: 1 ) from the ancestor having two PCs at opposite ends (in the marine lineage) and evolving to Auerbachia and Coccomyxa spp.; and 2) from the ancestor having its $\mathrm{PCs}$ at the anterior end (in the freshwater lineage) and evolving to Thelohanellus spp.

\section{Orientation of polar capsules to a plane of the suture}

The ancestor of all myxozoans had the PCs arranged in the plane perpendicular to the suture line. The ancestor of species of the freshwater lineage changed the position of the PCs and the primitive state reappeared independently several times in Sphaerospora spp., Hoferellus gilsoni and Myxobilatus gasterostei. The opposite situation can be seen in the marine clade: ancestors of several clades possessed primitive characters and the PC arrangement to a plane of the suture evolved in the recent ancestors of current species.

\section{Location of polar capsules and sporoplasm}

The primitive state is the position with PCs together at the anterior part of the spore with a posterior sporoplasm. PCs moved to the opposite end of the spore quite recently in evolution. This character evolved independently several times and it is common for species of four distinct phylogenetic groups including species of genera e.g. Myxidium, Zschokkella and Enteromyxum. 


\section{Shape of polar capsules}

The ancestor of all myxozoans had spherical or subspherical shape of PCs. Pyriform PCs evolved in the ancestors of the freshwater branch. Further, pyriform PCs appeared independently several times within the marine lineage.

\section{Position of tips of polar capsules}

The evolution of this character is linked to the arrangement of PCs within a spore. Divergent PCs evolved in parallel with the evolution of PCs in opposite positions and the history of their evolution is similar. One of few exceptions of this "co-evolution" is Tetracapsuloides bryosalmonae possessing divergent PCs arranged together in its fish malacospore.

\section{Character of polar filament}

The feature of having flattened polar filaments evolved only once and it is unique to species of the genus Sphaeromyxa.

\section{Number of sporoplasms}

The ancestral state is a single sporoplasm. A spore with two sporoplasms appeared in the common ancestor of basal sphaerosporids and multivalvulids and in several current Sphaerospora spp.

\section{Mucous envelope and membranaceous veil}

Most of myxozoans do not possess any of these spore characters. The presence of a mucous envelope or membranaceous veil appeared independently only a few times in terminal nodes and has no common evolutionary history.

\section{Vegetative stages}

The common ancestors of all myxozoans and the ancestor of species clustering in the marine lineage had small mono- or disporic plasmodia. Polysporic plasmodia probably evolved for the first time in the ancestors of the freshwater lineage and then twice independently in the marine lineage, whereas the reverse reduction of large plasmodia to the smaller ones occurred probably many times.

\section{Site of infection}

We analysed the site of infection as a two-state character defined as coelozoic and histozoic. It seems that there were only two main splits from coelozoic to histozoic site preference in the evolution of Myxozoa leading to: 1) Myxobolus/Henneguya species and 2) to the group containing multivalvulids and enteromyxids (including Ceratomyxa shasta in some analyses).

\section{Site specificity}

This multi-state character extends the previous one by more specific definition of the site of infection. The analysis of a particular site of infection suggested that the first myxozoans inhabited the excretory system. Later myxozoans then infected the gall bladder. The ancestors of several groups moved back from the gall bladder to the excretory system, intestine, or the muscles. Only the histozoic Myxobolus and Henneguya spp. show great variability in the site of infection. Many other phylogenetic groups include species with identical site specificities.

\section{Host}

The typical intermediate host of myxozoans is a fish. It is very likely that the ancestors of the main myxosporean groups infected the fish whereas several switches to other vertebrate taxa occurred later.

\section{Host environment}

The first myxozoan ancestor was a freshwater species and then myxozoans expanded to the marine environment. Thus, the ancestor of all Myxosporea was a marine species. Freshwater as the host environment occurred three times independently: by the ancestor of species of the freshwater lineage, by the ancestor of the freshwater basal sphaerosporid, and by Ceratomyxa shasta. Some freshwater species then re-entered the marine environment afterward. A typical returnee was the ancestor of species of the genus Sphaeromyxa, a diversified group of marine myxosporeans.

Tracing the evolution of particular characters facilitated the reconstruction of the evolution of the myxospores' morphology and bionomical characters (Figure 4). Based on our analyses, the ancestor of all myxozoans had a spore with two valves, was spherical in shape, had a width that was equal to its thickness, had no surface ridges and no projections, and had a straight suture line. Further, the spore had two subspherical and convergent PCs set in the plane perpendicular to the suture, with the PCs arranged at its anterior end. There was one posterior sporoplasm, a tubular polar filament, and no mucous envelope or membranaceous veil. The plasmodia were small and mono- or disporic, the site of infection was coelozoic, and it was a parasite of the excretory system of freshwater fishes. Furthermore, our analyses suggested some common trends in the myxosporean character evolution e.g.: i) myxosporeans with four PCs and two shell valves evolved only in the freshwater clade; ii) myxosporeans with four or more shell valves evolved only once in the marine clade; iii) myxosporeans with surface ridges or striations and caudal appendages evolved only in the freshwater clade; and iv) myxosporeans with PCs placed on opposite sides of the spindle or ellipsoid shaped spore evolved several times in both marine and freshwater clades. We found only one clear morphological synapomorphic character following the phylogenetic relationships of myxozoans. Although such incongruence between phylogeny and taxonomy is extreme among eukaryotes, a similar situation was documented in the Microsporidia [19]. Species of this fungal group reduced their body form to the plasmodial and spore stages similarly to the myxozoans. Further, and also similar to what occurs with the Myxozoa, the 
Microsporidian taxonomy based on the morphology of the spore is not congruent with the phylogeny of the group. The sole synapomorphic character is the polar filament. Its arrangement and flattened shape is unique for Sphaeromyxa spp. and it is one of the most important characters for the definition of the genus Sphaeromyxa. The other 19 characters that we traced were homoplastic. Nevertheless, bionomical characters (site of infection, site specificity, and host environment) correlate highly with the phylogeny. Although the myxospore's morphology generally does not correlate with the phylogeny, several morphological characters were found to correspond partially to the phylogeny. These myxospores characters are: the number of valves, the position of PCs relative to the sutural plane and the sporoplasm, the ratio of spore width to thickness, and the presence or absence of surface ridges. This is illustrated by the frequent presence of clusters of species with the same character features in the phylogenetic trees (additional file 4). Connection of these characters could be utilised in future taxonomic changes (taxon demises or the establishment of new genera or higher taxonomic levels) together with the combination of bionomical characters.

The main phylogenetic segregation according to the myxozoans hosts' habitat (freshwater and marine) were firstly documented by Kent et al. [20] and this trend is, in general, still valid with some exceptions. Our analyses also supported the site of infection and site specificity as unique characters that follow the phylogenetic pattern of many myxozoan species as published elsewhere $[21,10,22,11]$. However, there are some exceptions to this trend (e.g., Sinuolinea phylopteryxa, Myxidium hardella or Sphaerospora sp. EE2004). These latter parasites of renal tubules cluster among the gall bladder infecting species. Nevertheless, site specificity is still a common feature for many closely related species. Tracing the history of evolution of this character enables the assignment of the gall bladder site as a feature that evolved early in evolution. This feature persists among descendants and occurs among many current species. This means that all gall bladder infecting species with known SSU rDNA have ancestors with gall bladder site specificity except for the deepest ancestor of all myxozoans, which infected the excretory system. Furthermore, all species with different site specificity than the gall bladder evolved from ancestors infecting the gall bladder. This corresponds to Shulman's assumption [23] that the first myxosporean species was coelozoic and infected the gall bladder.

Spore projections or surface ridges can be considered as typical examples of homoplastic characters. They very likely have identical biological function and resulted from the convergent evolution in myxosporean species. Ridges probably help spores to float in freshwater, an assumption that is supported by the absence of such features in species from the marine environment, whose water has a higher density. The exception is the marine species Chloromyxum leydigi, which has a close phylogenetic relationship to the freshwater lineage. Surprisingly, no ridges appeared on the typical freshwater species-rich genus Myxobolus. However, floating in this case is probably facilitated by the development of caudal appendages. This character evolved independently many times during the diversification of numerous Myxobolus spp. and gave rise to the current Henneguya spp. Caudal appendages similar to those found in Henneguya spp. are also present in species of the genera Unicauda, Dicauda, Hennegoides, Tetrauronema, and Laterocaudata. Although the SSU rDNA sequences of these species are not available in GenBank, they would probably cluster within the Myxobolus clade and would be expected to have a similar evolutionary history as that of Henneguya spp.

Based upon our analysis caudal appendages should be considered as species characteristics but not generic characters. Suppression of the genus Henneguya can be expected as well as suppression of the genera Hennegoides, Tetrauronema, Laterocaudata, Dicauda and Unicauda after molecular data is obtained. Some taxonomic revisions, resulting from phylogenetic analyses, have been already carried out. Whipps et al. demised the genera Pentacapsula, Hexacapsula, and Septemcapsula and assigned their species to the genus Kudoa [14]. Further, Zschokkella mugilis was transferred to the genus Ellipsomyxa [24]. A radical taxonomic revision was also recently made by Gunter and Adlard, who demised the genus Leptotheca and transferred its species to the genera Ceratomyxa and Sphaerospora [25].

\section{Evolution of myxozoan morphotypes}

Our analysis has shown that the myxozoan common ancestor was a species with a spore morphology similar to that of current species of the genus Sphaerospora infecting the renal tubules of freshwater fishes. This supports the hypothesis of Jirků et al. that current myxozoans are derived from the Sphaerospora-type myxospore [26]. We confirmed that a group of basal sphaerosporids possesses features ancestral to present species. On the other hand, some Sphaerospora spp. with known SSU rDNA sequences branched at several distinct positions in the tree, which could be explained by a certain plasticity in the change of myxospores' shape that would best fit environmental factors. This supports the idea that the sphaerosporid morphotype might be a very successful spore design favoured by convergent evolution [10].

Based on our results, we propose several major trends in the evolution of myxospore morphotypes (Figure 4). The morphotype means a set of spore morphological characters typical for group of myxosporeans assigned to the 
same myxosporean genus (e.g. Myxidium or Myxobolus morphotype). According to Lom and Dyková there are about 60 described genera of Myxosporea [27], which ergo represent the same number of current spore morphotypes. A hypothetical ancestor of all species in the marine clade evolved from the ancestral sphaerosporid morphotype by an extension of the spore thickness, giving rise to the ceratomyxid morphotype. This morphotype is retained through the present as a group of species belonging to the genus Ceratomyxa. Intestinal Ceratomyxa shasta may have evolved either from a gall bladder infecting ancestral ceratomyxid (regardless of its uncertain phylogenetic position as a separate lineage in the marine clade [11]) or as a basal ceratomyxid [25]. We suggest a different hypothesis, that $C$. shasta shared a common ancestor with both enteromyxids and multivalvulids. After the switch from parasitism in the gall bladder to a site location in the intestine, $C$. shasta then separated from the enteromyxid and multivalvulid ancestor (dashed line in Figure 4). This possibility is the most parsimonious solution, with only one switch to different site of infection. This theory is also supported by our ML analysis (Figure 5). However, the analysis includes the limited number of Ceratomyxa sequences. The sphaerosporid morphotype very likely occurred by the ancestor of Parvicapsula spp. infecting the excretory system. Spores of Parvicapsula spp. are very similar to those of Sphaerospora spp. and basically represent a further step in the evolution of the sphaerosporid type of spore. The kudoid morphotype probably evolved by a duplication or multiplication of an ancestral extinct morphotype with an enteromyxid spore shape but with PCs close together. The PCs then independently moved to the opposite ends of the spore along with an extension of spore width several times in myxosporean evolution. This evolution gave rise to the species classified to the genera Myxidium, Zschokkella, Ellipsomyxa, Sphaeromyxa and Enteromyxum. Intestinal Enteromyxum spp. very likely did not evolved from the Myxidium or Zschokkella morphotype. Despite some similarities in the spore structure with Myxidium morphotype, Enteromyxum spp. probably evolved from the gall bladder infecting ceratomyxid ancestor. The morphotype with four polar capsules and two shell valves similar to that of existing Chloromyxum spp. probably evolved early after the separation of the marine lineage from the rest of the myxosporeans, including the freshwater lineage and marine Chloromyxum leydigi. We can hypothesize that the Chloromyxum morphotype was derived from an ancestral sphaerosporid by the duplication of polar capsules. Later in the evolution, myxospores then lost these additional two polar capsules, creating the predominant Myxobolus, Zschokkella and Myxidium morphotypes of the freshwater clade. Sphaeromyxa species had evolved by a change in the character of polar filament from the Myxidium ancestor, which then moved back to the marine environment.
Members of the most species-rich myxozoan genus, $M y x-$ obolus, probably evolved from the coelozoic ancestor infecting renal tubules. Myxobolus spp. invaded numerous host tissues and then segregated to numerous species during evolution. The origin of caudal appendages present on the Myxobolus morphotype led to the evolution of species classified in the genus Henneguya. Thelohanellus spp. evolved from the Myxobolus morphotype by the loss of one of its PCs. The same event probably occurred in both Auerbachia and Coccomyxa spp., which arose from the Myxidium morphotype in the marine clade. The origin of Myxobilatus and Hoferellus morphotypes with PCs placed perpendicular to the suture plane is another proof that the polar capsules freely change their position relative to the suture plane during evolution. This is typical for the origin of Sphaerospora species, which evolved convergently several times in the marine or freshwater clade. Gadimyxa spp. also differ from their parvicapsulid ancestor in the arrangement of PCs to a plane of the suture.

\section{Character evolution and phylogeny}

A summary of the spore morphology of selected myxosporeans of all genera of the main myxozoan phylogenetic groups is presented in the Figure 5. This summary demonstrates spore variability throughout the myxozoan phylogenetic tree, as well as the close relationship of species with very different spore structure and, vice versa, the distant relationships of species with very similar spore morphology. Figure 5 also gives the proportion of sequenced species (SSU rDNA) of each genus present in the particular clade relative to the number of all sequenced species of each genus and to overall number of described species of each genus. These numbers illustrate the lack of data in many genera. Ten out of about one hundred known species of Sphaerospora cluster into five different positions on the SSU rDNA tree. Holzer et al. interpreted this phenomenon to be a result of the convergent evolution of the primitive sphaerosporid spore shape [10]. The phylogenetic position of ninety Sphaerospora spp. is still not known. The extreme polyphyletic nature of species rich genera, such as Sphaerospora, $M y x-$ idium, Zschokkella and Chloromyxum, suggests that the many myxosporeans that have not yet been sequenced likely may cluster in any phylogenetic branch of the tree.

Significantly, molecular rDNA data are not able to resolve several evolutionary important nodes in the myxosporean phylogenetic tree [16]. The relationships of the main myxosporean phylogenetic groups of the marine clade, as defined in Fiala [11], are uncertain and depend on taxa sampling and the analysis performed. Tracing the evolutionary history of studied characters may enable resolution of some uncertain relationships. It has been proven that site of infection is a factor connecting many myxosporeans. Both Ceratomyxa shasta and Enteromyxum spp. 


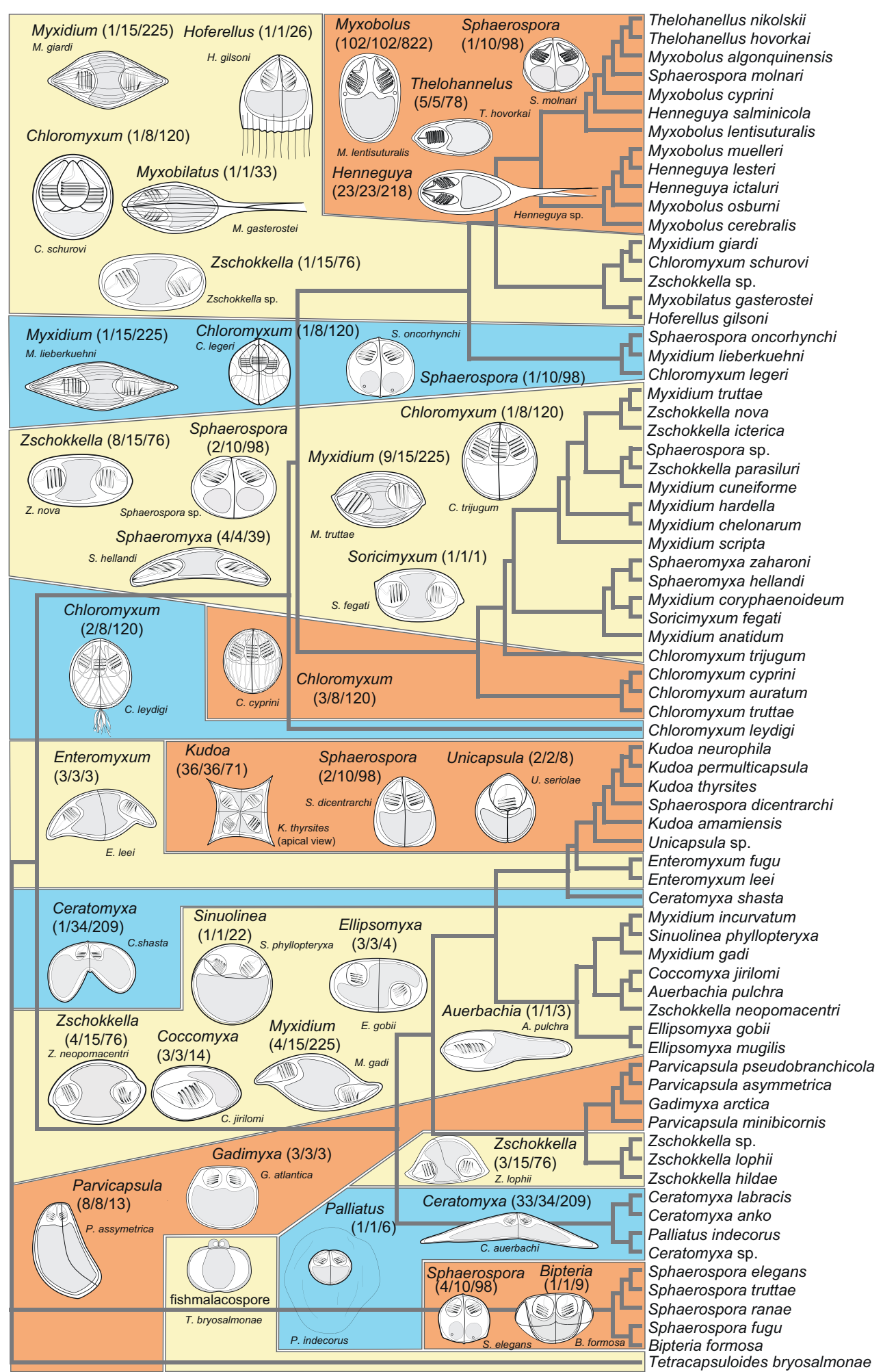

Figure 5 Spore morphology of selected myxozoans of all genera included in the main myxozoan phylogenetic groups. Maximum likelihood SSU rDNA tree of selected myxozoans covering the broad phylogeny of Myxozoa. The boxes join the myxozoans of main phylogenetic groups. Each box contains schematic drawings of the spores of representatives of all genera included in the group. Numbers in the brackets by the genera names show the number of sequenced species of the genus placed in the clade/number of overall sequenced species of the genus/number of described species of the genus. 
have uncertain position in the marine clade with low bootstrap supports [11] and identical sites of infection suggest their common evolution. The spore morphology of the hypothetical ancestor of enteromyxids and multivalvulids is likely very similar to C. shasta. However, this disagrees with a recent analysis of a large number of Ceratomyxa spp., wherein C. shasta branches as a basal taxon to all ceratomyxid species [28]. Nevertheless, Kudoa spp. + Enteromyxum spp. and C. shasta may constitute one histozoic clade in myxosporean evolution.

\section{Conclusions}

In this study we support rDNA based myxozoan phylogeny by sequencing and analyses of the protein coding gene EF2 and demonstrate the reliability of rDNA as a marker explaining myxozoan relationships. We propose the evolution of ancestral myxozoan morphotypes. The evolutionary history enables us to understand the evolution of modern species and supports some uncertain topologies resulting from the analyses of SSU rDNA data.

\section{Methods}

DNA isolation, PCR amplification, cloning and sequencing DNA was extracted from fresh myxospores using the DNeasy ${ }^{\text {Ta }}$ Tissue Kit (Qiagen, Germany) according to the manufacturer's protocol. EF2 gene was amplified using A1 (5'-GGNGCNGGNGARYTNCAYYTNGA-3) and A2 (5'-CCARTGRTCRAANACRCAYTGNGGRAA-3) primer set [29]. PCR was carried out in a $25 \mu \mathrm{l}$ reaction volume using $10 \mathrm{pmol}$ of each primer, $250 \mu \mathrm{M}$ of each $\mathrm{dNTP}$, and $2.5 \mu \mathrm{l} 10 \times$ PCR Buffer (Top-Bio, Czech Republic) and 1 unit of Taq-Purple polymerase (Top-Bio, Czech Republic). The reactions were run on a Tpersonal cycler (Biometra). Amplification consisted of initial denaturation at $95^{\circ} \mathrm{C}$ for $5 \mathrm{~min}$ and 35 cycles of $95^{\circ} \mathrm{C}$ for 1 min, $48^{\circ} \mathrm{C}$ for $1 \mathrm{~min}$ and $72^{\circ} \mathrm{C}$ for $1 \mathrm{~min}$, followed by 10 min final extension at $72^{\circ} \mathrm{C}$. The PCR products were isolated from the gel and cloned into $\mathrm{pCR}^{\circ} 2.1$-TOPO vector from the pDrive Cloning vector (Qiagen PCR Cloning Kit) and transformed into competent $E$. coli-strain XL-1. Both strands of clones were sequenced on ABI PRISM 3130xl automatic sequencer (Applied Biosystems).

\section{Alignments and phylogenetic analyses}

Seven alignments of EF2, SSU and LSU data with twelve myxosporeans were constructed. The cnidarian Aurelia sp. was set as outgroup. The alignments consisted of single gene data, concatenated SSU + LSU and concatenated $\mathrm{SSU}+\mathrm{LSU}+\mathrm{EF} 2$ data. EF2 gene was aligned as nucleotide sequence (EF2nt) as well as amino acid (EF2aa) sequence data. EF2nt were analysed with all three sites in codon, with the exclusion of the third site of codon and as codons. The alignments were aligned using MAFFT program [30] with E-INS-i method and gap opening penalty (-op) 5.0 and gap extension penalty (-ep) 0.0 . The alignments were visualised in SEAVIEW v. 3.2 [31].

Maximum parsimony (MP) analyses were performed in PAUP* 4.0b10 [32] using a starting tree build by heuristic search with random taxa addition, the ACCTRAN-option and the TBR swapping algorithm. Ts/Tv ratio of 1:2 was applied for rDNA data and the gaps were treated as missing data. Clade supports were estimated using 500 bootstrap-replicates with random sequence additions.

Maximum likelihood (ML) analyses of EF2aa and EF2nt as codon sequence type were performed in GARLI v. 0.96 [33]. Amino acid analysis was done using WAG model and F3x4 method of codon frequencies was chosen for the codon based analysis. All the other ML analyses were made in PAUP* 4.0b10. The best model of evolution was determined by the likelihood ratio test (LRT) implemented in the Modeltest 3.06 [34]. SSU, LSU and SSU + LSU analyses were performed under GTR $+\Gamma$ model. EF2nt analysis was performed under GTR $+\Gamma+$ I model. Bootstrap values were calculated by 500 bootstrap re-sampling.

Bayesian inference (BI) trees were constructed using MrBayes 3.0b4 [35]. Likelihood parameters that were set for the single gene analyses and for the data partitions of concatenated analyses correspond to the models used in ML. The number of Markov chain Monte Carlo (MCMC) generations was set to $1,000,000$ with every 100th tree saved (two independent runs of four simultaneous MCMC chains). AWTY system [36] was used to assess the length of MCMC run and Tracer v. 1.4.1 [37] was used to ascertain the length of burn-in periods.

\section{Morphological matrix and mapping of morphological characters}

Twenty morphological and bionomical characters were chosen for the analysis (Figure 3 ). The analysis includes (1) number of spore valves, (2) shape of spore, (3) ratio of dimensions of spore width to the thickness, (4) surface ridges and striations, (5) projections of the spore, (6) shape of suture line, (7) number of polar capsules, (8) orientation of polar capsules to a plane of suture, (9) location of polar capsules and sporoplasm, (10) shape of polar capsules, (11) position of tips of polar capsules, (12) character of polar filament, (13) number of sporoplasms, (14) mucous envelope, (15) membranaceous veil, (16) vegetative stages, (17) site of infection, (18) site specificity, (19) host, and (20) host environment. The SSU rDNA-based tree was chosen as the basis for reconstruction of ancestral states. The tree consists of 73 myxosporean species covering the myxosporean diversity of known SSU rDNA sequences and malacosporean Tetracapsuloides bryosalmonae as the outgroup. The tree was constructed using ML with GTR $+\Gamma+\mathrm{I}$ model of evolution in PAUP* based on the alignment computed by MAFFT with parameters described above. 
Tree branches with uncertain phylogenetic relationships unstable positions in the tree as described in Fiala [11] were collapsed in their nodes resulting into polytomy for more accurate reconstruction of myxozoan phylogenetic relationships. History of character change was traced using the program Mesquite 2.5 [38]. Reconstruction of character states at ancestral nodes was done by likelihood method. We used Markov k-state 1 parameter model with the single parameter (the rate of change) [39]. Any particular change from one state to another is equally probable within this model.

\section{Additional material}

Additional file 1: Alternative EF2 tree topologies. Maximum likelihood tree topologies of a) EF2 nucleotide sequence based analysis (-In = 6943.4742, GTR $+\Gamma+$ I model, $\alpha=1.0804$, pinvar $=0.2586)$, b) EF2 nucleotide sequence based analysis with exclusion of the third site of codon $(-\ln =3280.0776$, GTR $+\Gamma+$ I model, $\alpha=1.6415$, pinvar $=0.4103)$, c) EF2 codon based analysis ( $-\ln =6513.1449$, state frequencies $\mathrm{f3} \times 4$ method). Bootstrap values (> 50\%) are indicated at the nodes. Cnidarian Aurelia sp. was set as outgroup sequence. Scale bar is given under the tree

Additional file 2: SSU and LSU rDNA tree topologies. Maximum likelihood trees constructed under a) SSU rDNA data ( In $=6127.1469$, GTR $+\Gamma$ model, $\alpha=0.3861)$ and b) LSU rDNA data $(-\ln =8824.2894$, $\mathrm{GTR}+\Gamma$ model, $\alpha=0.317$ ). Bootstrap values (> 50\%) are indicated at the nodes. Cnidarian Aurelia sp. was set as outgroup sequence. Scale bar is given under the tree.

Additional file 3: Combined SSU, LSU and EF2 tree topologies. The phylogenetic trees based on Bayesian inference of combined SSU rDNA + LSU rDNA + EF2 data. a) EF2 as amino acid sequence data, b) EF2 as nucleotide sequence data, c) EF2 as nucleotide data with exclusion of the third site of codon. The Bayesian posterior probabilities are indicated at the nodes. Cnidarian Aurelia sp. was set as outgroup sequence. Scale bar is given under the tree.

Additional file 4: Evolution of particular myxozoan characters. Twenty cladograms show the evolutionary history of all morphological and bionomical characters under study. The balls by the nodes represent proportional likelihoods of character states. See legends for colours of particular character states.

\section{Acknowledgements}

We are grateful to Prof. Iva Dyková and to Dr. Jiří Lom for helpful comments. We would like to thank also to Drs. Isaure De Buron and Vincent Connors for English corrections of the manuscript. This work was supported by Grant Agency of the Academy of Sciences of the Czech Republic (KJB600960701), by the Grant Agency of the Czech Republic (524/03/H133), by the research projects of the Institute of Parasitology, Biology Centre of the ASCR (Z60220518 and LC522) and by the research project of the Faculty of Sciences, University of South Bohemia (MSM6007665801)

\section{Author details}

${ }^{1}$ Institute of Parasitology, Biology Centre, Academy of Sciences of the Czech Republic, Branišovská 31, 37005 České Budějovice, Czech Republic. ${ }^{2}$ Faculty of Science, University of South Bohemia, Branišovská 31, 37005 České Budějovice, Czech Republic.

\section{Authors' contributions}

IF carried out the DNA sequence studies, performed phylogenetic analyses, performed the analyses of character evolution and drafted the manuscript. PB participated on DNA sequenced studies, participated on compiling of character matrix and helped to draft the manuscript. Both authors approved the final manuscript.

Received: 30 April 2010 Accepted: 28 July 2010 Published: 28 July 2010

\section{References}

1. Monteiro AS, Okamura B, Holland PWH: Orphan worm finds a home: Buddenbrockia is a myxozoan. Molecular Biology and Evolution 2002, 19(6):968-971.

2. Smothers JF, Vondohlen CD, Smith LH, Spall RD: Molecular evidence that the myxozoan protists are metazoans. Science 1994, 265(5179):1719-1721.

3. Stolc A: Actinomyxidies, nouveau groupe de Mesozoaires parent des Myxosporidies. Bull Intl Acad Sci Boheme 1899, 22:1-12.

4. Weill $R$ : $L$ 'interpretation des Cnidosporidies et la valeur taxonomique de leur cnidome. Leur cycle comparé ii la phase larvaire des Narcomeduses Cuninides. Travaux de la Station Zoologique de Wimereaux 1938, 13:727-744.

5. Siddall ME, Martin DS, Bridge D, Desser SS, Cone DK: The demise of a phylum of protists: Phylogeny of myxozoa and other parasitic cnidaria. Journal of Parasitology 1995, 81(6):961-967.

6. Hanelt B, VanSchyndel D, Adema CM, Lewis LA, Loker ES: The phylogenetic position of Rhopalura ophiocomae (Orthonectida) based on $18 \mathrm{~S}$ ribosomal DNA sequence analysis. Molecular Biology and Evolution 1996, 13(9):1187-1191

7. Siddall ME, Whiting MF: Long-branch abstractions. Cladistics-the International Journal of the Willi Hennig Society 1999, 15(1):9-24.

8. Jimenez-Guri E, Philippe H, Okamura B, Holland PWH: Buddenbrockia is a Cnidarian worm. Science 2007, 317(5834):116-118.

9. Kent ML, Andree KB, Bartholomew JL, El-Matbouli M, Desser SS, Devlin RH Feist SW, Hedrick RP, Hoffmann RW, Khattra J, et al: Recent advances in our knowledge of the Myxozoa. Journal of Eukaryotic Microbiology 2001, 48(4):395-413.

10. Holzer AS, Sommerville C, Wootten R: Molecular relationships and phylogeny in a community of myxosporeans and actinosporeans based on their 18 S rDNA sequences. International Journal for Parasitology 2004, 34(10):1099-1111.

11. Fiala I: The phylogeny of Myxosporea (Myxozoa) based on small subunit ribosomal RNA gene analysis. International Journal for Parasitology 2006, 36(14):1521-1534.

12. Nei $M$, Kumar $S$, Takahashi $K$ : The optimization principle in phylogenetic analysis tends to give incorrect topologies when the number of nucleotides or amino acids used is small. Proceedings of the National Academy of Sciences of the United States of America 1998, 95(21):12390-12397.

13. Philippe $H$, Germot A: Phylogeny of eukaryotes based on ribosomal RNA: Long-branch attraction and models of sequence evolution. Molecular Biology and Evolution 2000, 17(5):830-834.

14. Whipps CM, Grossel G, Adlard RD, Yokoyama H, Bryant MS, Munday BL, Kent ML: Phylogeny of the multivalvulidae (Myxozoa: Myxosporea) based on comparative ribosomal DNA sequence analysis. Journal of Parasitology 2004, 90(3):618-622.

15. Whipps CM, Kent ML: Phylogeography of the cosmopolitan marine parasite Kudoa thyrsites (Myxozoa: Myxosporea). Journal of Eukaryotic Microbiology 2006, 53(5):364-373.

16. Bartosova P, Fiala I, Hypsa V: Concatenated SSU and LSU rDNA data confirm the main evolutionary trends within myxosporeans (Myxozoa: Myxosporea) and provide an effective tool for their molecular phylogenetics. Molecular Phylogenetics and Evolution 2009, 53(1):81-93.

17. Holzer AS, Wootten R, Sommerville $C$ : The secondary structure of the unusually long $18 \mathrm{~S}$ ribosomal RNA of the myxozoan Sphaerospora truttae and structural evolutionary trends in the Myxozoa. International Journal for Parasitology 2007, 37(11):1281-1295.

18. Marotta R, Ferraguti M, Erséus C, Gustavsson LM: Combined-data phylogenetics and character evolution of Clitellata (Annelida) using $18 \mathrm{~S}$ rDNA and morphology. Zoological Journal of the Linnean Society 2008, 154:1-26.

19. Vossbrinck CR, Debrunner-Vossbrinck BA: Molecular phylogeny of the Microsporidia: ecological, ultrastructural and taxonomic considerations. Folia Parasitologica 2005, 52(1-2):131-142. 
20. Kent ML, Khattra J, Hedrick RP, Devlin RH: Tetracapsula renicola n. sp (Myxozoa: Saccosporidae); The PKX myxozoan - The cause of proliferative kidney disease of salmonid fishes. Journal of Parasitology 2000, 86(1):103-111.

21. Andree KB, El-Matbouli M, Hoffman RW, Hedrick RP: Comparison of $18 \mathrm{~S}$ and ITS-1 rDNA sequences of selected geographic isolates of Myxobolus cerebralis. International Journal for Parasitology 1999, 29(5):771-775.

22. Eszterbauer E: Genetic relationship among gill-infecting Myxobolus species (Myxosporea) of cyprinids: molecular evidence of importance of tissue-specificity. Diseases of Aquatic Organisms 2004, 58(1):35-40.

23. Shulman SS: Myxosporidia of the fauna of the USSR. Moscow: Nauka 1966.

24. Koie M, Karlsbakk E: Ellipsomyxa syngnathi sp n. (Myxozoa, Myxosporea) in the pipefish Syngnathus typhle and S. rostellatus (Teleostei, Syngnathidae) from Denmark. Parasitology Research 2009, 105(6):1611-1616.

25. Gunter N, Adlard R: The demise of Leptotheca Thelohan, 1895 (Myxozoa: Myxosporea: (eratomyxidae) and assignment of its species to Ceratomyxa Thelohan, 1892 (Myxosporea: Ceratomyxidae), Ellipsomyxa Koie, 2003 (Myxosporea: Ceratomyxidae), Myxobolus Butschli, 1882 and Sphaerospora Thelohan, 1892 (Myxosporea: Sphaerosporidae). Systematic Parasitology 2010, 75(2):81-104.

26. Jirku M, Fiala I, Modry D: Tracing the genus Sphaerospora: rediscovery, redescription and phylogeny of the Sphaerospora ranae (Morelle, 1929) n. comb. (Myxosporea, Sphaerosporidae), with emendation of the genus Sphaerospora. Parasitology 2007, 134:1727-1739.

27. Lom J, Dykova I: Myxozoan genera: definition and notes on taxonomy, life-cycle terminology and pathogenic species. Folia Parasitologica 2006, 53(1):1-36.

28. Gunter NL, Whipps CM, Adlard RD: Ceratomyxa (Myxozoa: Bivalvulida): Robust taxon or genus of convenience? International Journal for Parasitology 2009, 39(12):1395-1405.

29. Hashimoto T, Nakamura Y, Kamaishi T, Nakamura F, Adachi J, Okamoto K, Hasegawa M: Phylogenetic place of mitochondrial-lacking protozoan, Giardia lamblia inferred from amino-acid sequences of elongation factor 2. Molecular Biology and Evolution 1995, 12(5):782-793.

30. Katoh K, Misawa K, Kuma K, Miyata T: MAFFT: a novel method for rapid multiple sequence alignment based on fast Fourier transform. Nucleic Acids Research 2002, 30(14):3059-3066

31. Galtier N, Gouy M, Gautier C: SEAVIEW and PHYLO_WIN: Two graphic tools for sequence alignment and molecular phylogeny. Computer Applications in the Biosciences 1996, 12(6):543-548.

32. Swofford DL: PAUP*: Phylogenetic Analysis Using Parsimony (*and other methods), Version 4.0b8. Sunderland, Massachusetts: Sinauer Associates 2001.

33. Zwickl DJ: Genetic algorithm approaches for the phylogenetic analysis of large biological sequence datasets under the maximum likelihood criterion. The University of Texas at Austin 2006.

34. Posada D, Crandall KA: MODELTEST: testing the model of DNA substitution. Bioinformatics 1998, 14(9):817-818.

35. Ronquist F, Huelsenbeck JP: MrBayes 3: Bayesian phylogenetic inference under mixed models. Bioinformatics 2003, 19(12):1572-1574.

36. AWTY: A system for graphical exploration of MCMC convergence in Bayesian phylogenetic inference. [http://ceb.csit.fsu.edu/awty].

37. Rambaut A, Drummond AJ: Tracer v1.4. 2007 [http://beast.bio.ed.ac.uk/ Tracer].

38. Mesquite: a modular system for evolutionary analysis. Version 2.5. [http://mesquiteproject.org].

39. Schluter D, Price $T$, Mooers AO, Ludwig D: Likelihood of ancestor states in adaptive radiation. Evolution 1997, 51(6):1699-1711.

doi:10.1186/1471-2148-10-228

Cite this article as: Fiala and Bartošová: History of myxozoan character evolution on the basis of rDNA and EF-2 data. BMC Evolutionary Biology $201010: 228$

\section{Submit your next manuscript to BioMed Central and take full advantage of:}

- Convenient online submission

- Thorough peer review

- No space constraints or color figure charges

- Immediate publication on acceptance

- Inclusion in PubMed, CAS, Scopus and Google Scholar

- Research which is freely available for redistribution

Submit your manuscript at www.biomedcentral.com/submit
C Biomed Central 